\title{
Estrogen treatment decreases matrix metalloproteinase (MMP)-9 in autoimmune demyelinating disease through estrogen receptor alpha $(E R \alpha)$
}

\author{
Stefan M Gold ${ }^{1,2}$, Manda V Sasidhar ${ }^{1}$, Laurie B Morales ${ }^{1}$, Sienmi Du ${ }^{1}$, Nancy L Sicotte ${ }^{1}$, Seema K Tiwari-Woodruff ${ }^{1}$ \\ and Rhonda R Voskuhl ${ }^{1}$
}

Matrix metalloproteinases (MMPs) have a crucial function in migration of inflammatory cells into the central nervous system (CNS). Levels of MMP-9 are elevated in multiple sclerosis (MS) and predict the occurrence of new active lesions on magnetic resonance imaging (MRI). This translational study aims to determine whether in vivo treatment with the pregnancy hormone estriol affects MMP-9 levels from immune cells in patients with MS and mice with experimental autoimmune encephalomyelitis (EAE). Peripheral blood mononuclear cells (PBMCs) collected from three female MS patients treated with estriol and splenocytes from EAE mice treated with estriol, estrogen receptor (ER) $\alpha$ ligand, ER $\beta$ ligand or vehicle were stimulated ex vivo and analyzed for levels of MMP-9. Markers of CNS infiltration were assessed using MRI in patients and immunohistochemistry in mice. Supernatants from PBMCs obtained during estriol treatment in female MS patients showed significantly decreased MMP-9 compared with pretreatment. Decreases in MMP-9 coincided with a decrease in enhancing lesion volume on MRI. Estriol treatment of mice with EAE reduced MMP-9 in supernatants from autoantigen-stimulated splenocytes, coinciding with decreased CNS infiltration by T cells and monocytes. Experiments with selective ER ligands showed that this effect was mediated through ER $\alpha$. In conclusion, estriol acting through $\mathrm{ER} \alpha$ to reduce MMP-9 from immune cells is one mechanism potentially underlying the estriol-mediated reduction in enhancing lesions in MS and inflammatory lesions in EAE.

Laboratory Investigation (2009) 89, 1076-1083; doi:10.1038/labinvest.2009.79; published online 10 August 2009

KEYWORDS: estriol; experimental autoimmune encephalomyelitis; leukocyte transmigration; multiple sclerosis

Matrix metalloproteinases (MMPs), particularly MMP-9, have been implicated in the disruption of the blood-brain barrier (BBB) in multiple sclerosis (MS) because the capacity of $\mathrm{T}$ cells and monocytes to transmigrate into the central nervous system (CNS) has been shown to be dependent on the activity of MMP-9. Levels of MMPs have been found to be upregulated in several inflammatory CNS diseases ${ }^{1}$ including MS in which autopsy studies have shown that MMP-9 is increased in macrophages and lymphocytes within perivascular cuffs. ${ }^{2}$ Furthermore, serum and CSF levels of MMP9 are higher in relapsing remitting multiple sclerosis (RRMS), particularly in patients during relapse and in those with enhancing lesions on magnetic resonance imaging (MRI). ${ }^{3-6}$ Increased MMP-9 serum levels have also been found in patients with clinically isolated syndrome and MMP-9 levels further increased in patients who developed clinically definite MS compared with stable levels in patients who did not convert. $^{7}$

During pregnancy, relapse rates in MS are decreased by approximately $80 \%$ during the last trimester, ${ }^{8}$ a time when the pregnancy hormone estriol reaches its highest levels. Studies in experimental autoimmune encephalomyelitis (EAE), the animal model of MS, have shown that estriol treatment ameliorates disease ${ }^{9,10}$ and decreases inflammatory lesions in the spinal cord. ${ }^{11}$ Anti-inflammatory effects of estrogens are complex and include effects on chemokines, cytokines, dendritic cell function, and $\mathrm{T}$ regulatory cell subpopulations. ${ }^{12}$ Estriol has been shown to downregulate TNF- $\alpha,{ }^{10}$ a cytokine known to activate MMP-9. ${ }^{13}$ Consistent with the anti-inflammatory effects of estriol in EAE, a recent

\footnotetext{
${ }^{1}$ Multiple Sclerosis Program, Department of Neurology, David Geffen School of Medicine at UCLA, Los Angeles, CA, USA and ${ }^{2}$ Cousins Center, Semel Institute for Neuroscience, David Geffen School of Medicine at UCLA, Los Angeles, CA, USA

Correspondence: Professor RR Voskuhl, MD, Department of Neurology, University of California, Los Angeles, 635 Charles Young Drive South, NRB1 Room 475D, Los Angeles, CA 90095, USA. E-mail: rvoskuhl@ucla.edu

Received 11 February 2009; revised 18 June 2009; accepted 7 July 2009
} 
pilot study of estriol treatment in female MS patients showed decreased levels of TNF- $\alpha$ from peripheral blood mononuclear cells $(\mathrm{PBMCs})^{14}$ and decreases in the number and volume of gadolinium-enhancing lesions on MRI. ${ }^{15}$ Finally, $\mathrm{T}$ cells obtained from MS patients, which were treated with pregnancy levels of estriol, showed decreased MMP-9 levels and lower migratory capacity in vitro. ${ }^{16}$ What remains unclear is whether MMP-9 downregulation occurs in vivo when estriol is administered at a pregnancy dose and if this is related to decreases in markers of CNS infiltration during autoimmune demyelinating disease. In addition, it is unclear whether this effect is mediated through estrogen receptor (ER) $\alpha$ or $\operatorname{ER} \beta$.

\section{MATERIALS AND METHODS Female RRMS Patients}

Peripheral blood mononuclear cells were obtained from three premenopausal female patients with clinically definite RRMS who had participated in our pilot trial of oral estriol treatment with $8 \mathrm{mg} /$ day for a duration of 6 months. ${ }^{15}$ The study was approved by the UCLA Human Subjects Protection Committee, and informed consent was obtained. The dose used has been shown to yield estriol levels in the blood that approximated 6-month pregnancy levels in humans. ${ }^{15}$ PBMCs were isolated and cryopreserved at three time points: before estriol treatment, at the end of the 6-month estriol treatment period and 3 months after the cessation of treatment.

\section{PBMC Cultures, MMP Measurement and Intracellular MMP-9 Staining}

PBMCs were cultured at $1 \times 10^{5}$ per well with PHA $(5 \mu \mathrm{g} / \mathrm{ml}$; Sigma-Aldrich, at $37^{\circ} \mathrm{C}, 5 \% \mathrm{CO}_{2}$ ) and supernatants were collected after $48 \mathrm{~h}$. Culture supernatants were assayed for levels of MMP-9, its inhibitor TIMP-1, MMP-2 and its inhibitor TIMP-2 using SearchLight multiplex assays. To assess functional proteolytic activity of MMP-9 in the PBMC cell culture supernatants, zymography assays were used. Supernatants were diluted $1 / 1$ in $2 \times$ zymogram sample dilution buffer (NOVEX, San Diego, CA, USA). Then, $10 \mu$ l of diluted sample was loaded onto a precast $10 \%$ Tris/glycine gel with $0.1 \%$ gelatin incorporated as substrate (NOVEX). Gels were electrophoresed at $125 \mathrm{~V}$ for $90 \mathrm{~min}$ and then renatured for $30 \mathrm{~min}$ in $1 \times$ renaturing buffer (NOVEX) at room temperature. This was followed by incubation in $1 \times$ developing buffer at $37^{\circ} \mathrm{C}$ for $18 \mathrm{~h}$. Gels were stained in $0.5 \%$ Coomassie blue R-250 (Bio-Rad, Hercules, CA, USA) dissolved in 30\% methanol $/ 10 \%$ acetic acid and destained in the same solution without dye. Gels were scanned using an Epson 4870 scanner, and converted to grayscale in Adobe Photoshop. Band intensities were quantified by ImageJ software using the semiautomated Gel Analysis Tool.

For intracellular MMP-9 staining, PBMCs were stimulated with $5 \mu \mathrm{g} / \mathrm{ml}$ PHA for $24 \mathrm{~h}$ in the presence of monensin $(2 \mu \mathrm{M})$ to allow for intracellular accumulation. Cells were washed and stained with conjugated surface antibodies for CD3 (APC), CD14 (APC), CD16 (PerCp) and CD56 (PE) (Biolegend, San Diego, CA, USA), washed, fixed and permeabilized with Cytofix/Cytoperm solution (BD PharMingen, San Diego, CA). Then, cells were stained with FITClabeled Ab specific for MMP-9 or isotype control (R\&D Systems, Minneapolis, MN, USA), washed and resuspended for FACS analysis on a FACSCalibur instrument (BD Biosciences, San Diego, CA) using CellQuest software (BD Biosciences).

\section{Brain MRI and Lesion Quantification}

MRI scans obtained in RRMS patients in the estriol trial were analyzed at three time points: before estriol treatment, in the last month of estriol treatment (month 6) and 3 months after the cessation of treatment. T1-weighted scans with and without gadolinium $(\mathrm{Gd})$, were performed on a 1.5T G.E. scanner. The volume of gadolinium-enhancing lesions was determined using a semi-automated threshold based technique (Display; Montreal Neurological Institute) as described. ${ }^{15}$

\section{Animals}

Female C57BL/6 mice, 8 weeks of age, were purchased from Taconic (Germantown, NY, USA). Animals were housed under guidelines set by the National Institutes of Health, and experiments were conducted in accordance with the University of California, Los Angeles, Chancellor's Animal Research Committee and the Public Service Policy on Humane Care and Use of Laboratory Animals.

\section{Active EAE Induction}

Active EAE induction ensued with subcutaneous injection of an emulsion containing the autoantigen, myelin oligodendrocyte glycoprotein (MOG) peptide, amino acids 35-55 (300 $\mu \mathrm{g} / \mathrm{mouse})$ and Mycobacterium tuberculosis (500 $\mu \mathrm{g} /$ mouse) in complete Freund's adjuvant. Mice were monitored daily for EAE disease severity using the standard EAE grading scale as described. ${ }^{17}$

\section{Treatment and Reagents}

Gonadally intact female mice were treated with 60-day continuous release estriol pellets $(5 \mathrm{mg}$ ) or placebo pellets (Innovative Research of America, Sarasota, FL, USA) beginning 7 days before EAE induction. This dose has been shown earlier to yield circulating estriol levels that approximated levels of estriol during late natural pregnancy in a mouse. ${ }^{9}$

Additional sets of mice were anesthetized with isoflurane, ovariectomized and allowed to recuperate for 10 days. Animals were then treated with a selective ER $\alpha$ ligand propyl pyrazole triol (PPT) at $10 \mathrm{mg} / \mathrm{kg}$ per day, selective $\mathrm{ER} \beta$ ligand diarylpropionitrile (DPN) at $8 \mathrm{mg} / \mathrm{kg}$ per day, or vehicle beginning 7 days before EAE induction and throughout the entire disease duration by daily s.c. injections. These doses of ER ligands have been shown earlier to yield expected effects on a positive control tissue (uterus). ${ }^{18}$ On day $35-40$ 
after disease induction, animals were sacrificed, spleens removed and animals perfused for immunohistochemistry as described. ${ }^{18}$

\section{Splenocyte Cultures and MMP-9 Measurement}

Splenocytes were isolated and stimulated in vitro with autoantigen, MOG peptide $35-55$, at $25 \mu \mathrm{g} / \mathrm{ml}$ for $48 \mathrm{~h}$. MMP-9 protein levels were assayed in culture supernatants using SearchLight multiplex assays (anti-mouse antibodies). MMP-9 amount in the splenocyte culture supernatants was assessed with zymography as described above.

\section{Immunohistochemistry}

Spinal cords were isolated from perfused mice and processed as described. ${ }^{18}$ Free-floating cross sections $(25 \mu \mathrm{m}$ thick) were cut with a sliding microtome and collected serially in PBS. Consecutive sections were examined by immunohistochemistry. The following primary antibodies were used for $\mathrm{T}$ cells (CD3, 1:500, BD Pharmingen), and macrophages (Mac3, 1:300, BD Pharmingen) and a nuclear stain DAPI ( $2 \mathrm{ng} / \mathrm{ml}$; Molecular Probes). Mounted and stained sections were examined and photographed using a confocal microscope (Olympus Spin disc confocal microscope, Japan). To quantify immunostaining results, sections from thoracic spinal cord levels T1-T5 were examined, three from each mouse, with $n=3$ mice per treatment group. Cell numbers were quantified (at $\times 40$ magnification) by counting the $\mathrm{CD} 3+/ \mathrm{DAPI}+$ and Mac3 +/DAPI + cells per $100 \mu \mathrm{m}^{2}$ in the dorsal column of the spinal cord by a masked observer.

\section{Statistical Analysis}

MMP levels were compared in RRMS patients from pretreatment to treatment period using paired $t$-tests. MMP-9 levels between estriol and vehicle-treated mice with EAE were compared using independent $t$-tests. MMP-9 levels in groups of mice treated with ER $\alpha$ ligand, ER $\beta$ ligand or vehicle were compared using one-way ANOVA with Bonferroni-adjusted post tests. Group differences in EAE scores were tested using repeated measures mixed model ANOVA with Bonferroniadjusted post tests. Group differences in T cell and macrophage counts in immunohistochemistry were tested using one-way ANOVA with Newman-Keuls multiple comparison tests. A value of $P<0.05$ was considered statistically significant. All analyses were computed using GraphPad Prism Software 4.0 for Macintosh.

\section{RESULTS \\ MMP-9, but not MMP-2, is Decreased by Estriol Treatment in RRMS}

MMP-9 levels and activity were decreased in RRMS patients treated with pregnancy levels of estriol. Zymography, an enzymatic activity-based assay, showed decreased amounts of MMP-9 in supernatants from ex vivo stimulated PBMCs obtained from patients during estriol treatment compared with pre- and 3 months post treatment (Figure 1a). In addition, during treatment, a significant decrease in MMP-9/ TIMP-1 ratio $(P=0.04$; Figure $1 b)$ was observed in supernatants from PBMC cultures. This was driven by significant decreases in MMP-9 $(P=0.02$; Figure 1c), whereas TIMP-1 was not altered $(P=0.76$; Figure 1d). No significant changes were observed in MMP-2/TIMP2 ratio $(P=0.51$; Figure 1e), MMP-2 $(P=0.81$; Figure 1f $)$ or TIMP-2 levels $(P=0.60$; Figure 1g).

Within the same three RRMS patients, changes in MMP-9 were accompanied by a simultaneous decrease in enhancing $(\mathrm{Gd}+)$ lesion volume during estriol treatment as compared to before treatment (Figure 1h), consistent with our earlier publication showing that estriol treatment significantly decreases Gd + lesions. ${ }^{15}$ Representative scans from one subject at baseline and during treatment (Figure 1i) show resolution of an enhancing lesion (white arrow). Flow cytometric analysis revealed that $\mathrm{T}$ cells were a major source of MMP-9 in PBMCs stimulated with PHA (Figure 1j). This, however, does not rule out MMP-9 production by non-T cells using other stimulation conditions.

\section{Estriol Treatment Decreases MMP-9 in EAE}

Next, we ascertained whether we could replicate the observation from the estriol trial in female RRMS patients by administering estriol treatment to female C57BL/6 mice with EAE. Both zymography $(P=0.04$; Figure $2 \mathrm{a}-\mathrm{b})$ and protein measurement by SearchLight assays $(P<0.001$; Figure $2 \mathrm{c})$ showed decreased MMP-9 in supernatants obtained from autoantigen-stimulated splenocytes of estriol-treated EAE mice compared to vehicle treated. Clinically, estriol treatment significantly ameliorated disease severity $(P<0.0001$; Figure 2d). Immunohistochemistry showed that estriol treatment decreased infiltration by $\mathrm{T}$ cells $(P=0.001$; Figure $2 \mathrm{e}$, representative images shown in Figure $2 \mathrm{f}$ and $\mathrm{g}$ ) and macrophages $(P=0.04$; Figure 2e, representative images shown in Figure $2 \mathrm{~h}$ and $\mathrm{i})$ in the spinal cord.

Figure 1 MMP-9 regulation by estriol in multiple sclerosis. Six months of estriol treatment reduced MMP-9 bioactivity in peripheral blood mononuclear cell (PBMC) culture supernatants (PHA stimulated) as measured by zymography compared with pretreatment (pre) and 3 month after treatment cessation (post) in three female patients with relapsing-remitting MS (a). Estriol significantly decreased MMP-9/TIMP1 ratio (b). This effect was driven by decreases in MMP-9 levels (c), whereas estriol had no effect on TIMP1 (d). No significant changes were observed on MMP-2/TIMP2 ratio (e), MMP-2 (f) or TIMP2 (g). Decreased volumes of gadolinium-enhancing lesions on MRI occurred during estriol treatment (h). Representative scans from one subject show resolution of an enhancing lesion (i, white arrow). Intracellular staining using flow cytometry indicated that T cells (upper right quadrant) were a major source of MMP-9 in PHA activated PBMCs (j). 

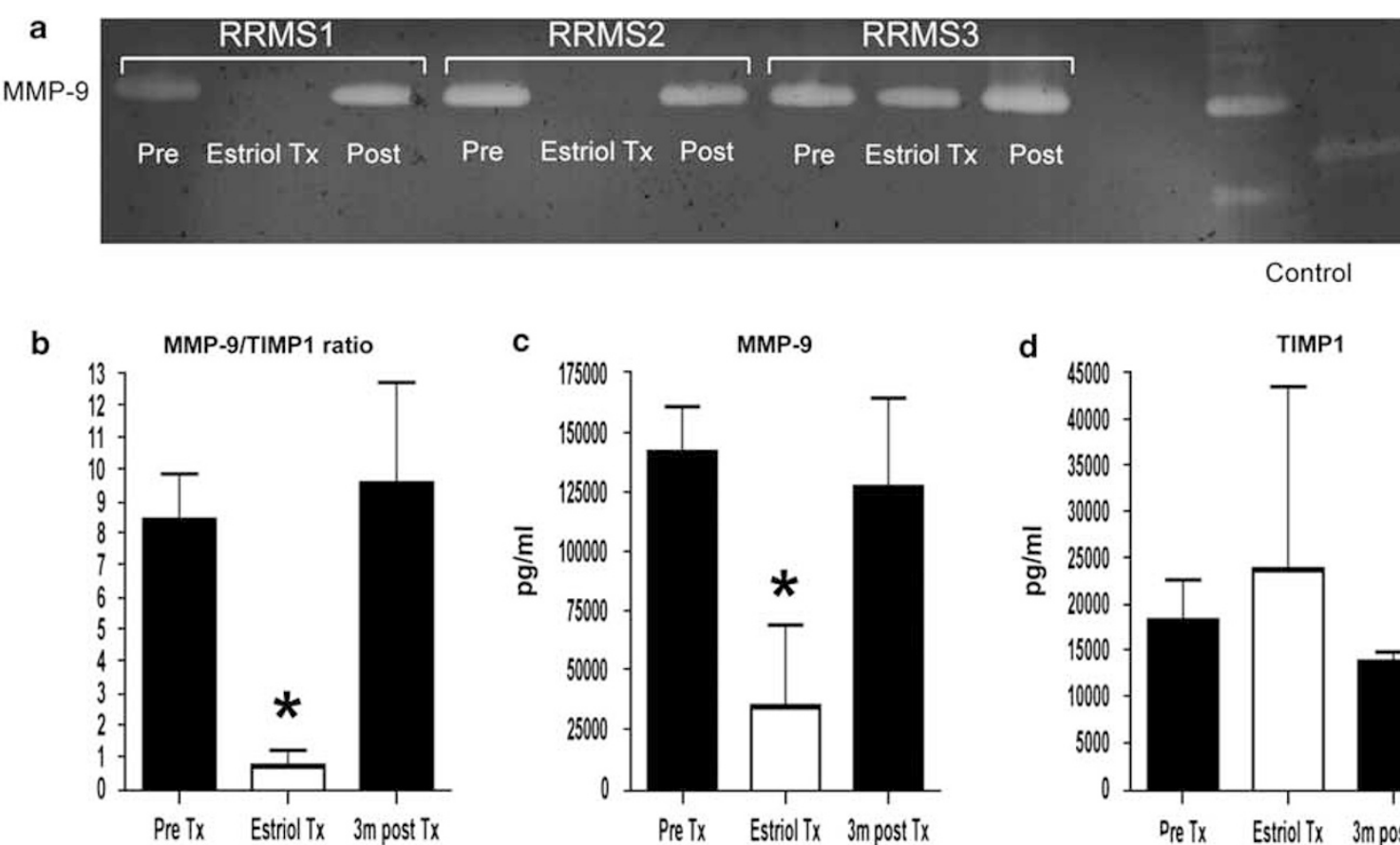

Control

e
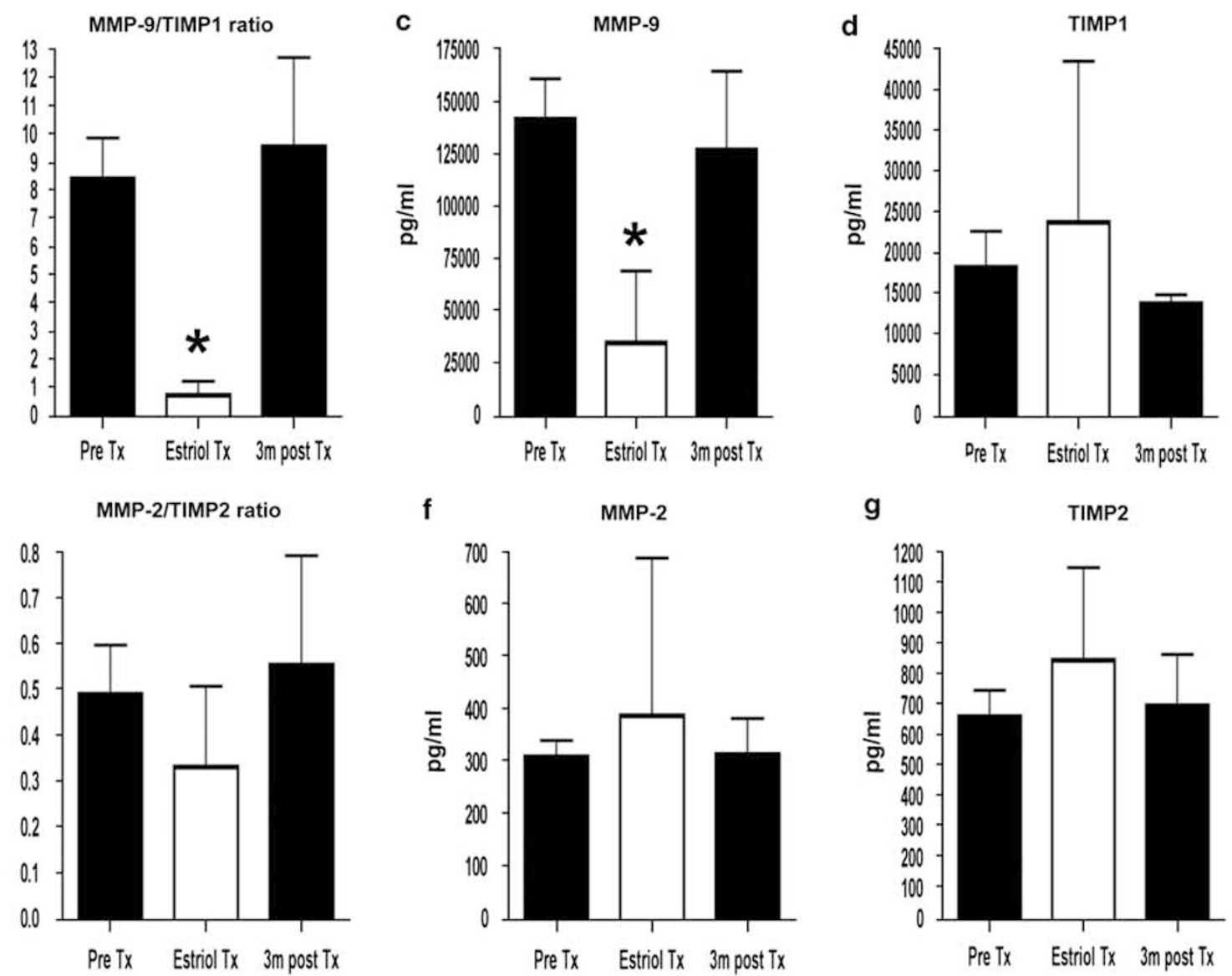

h

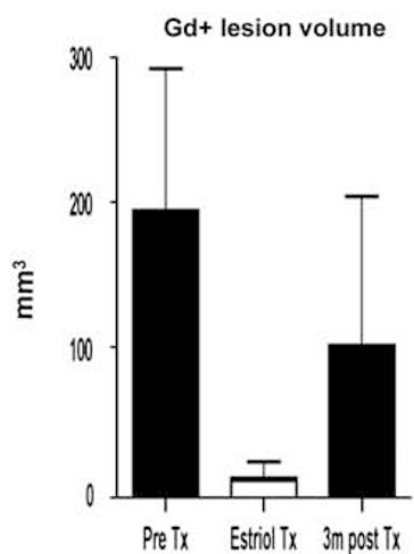

i

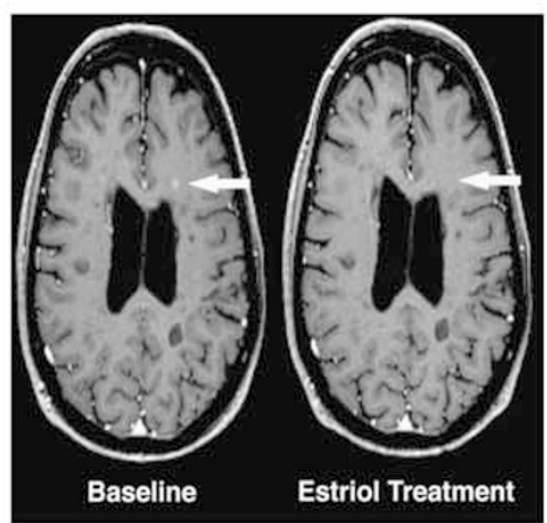

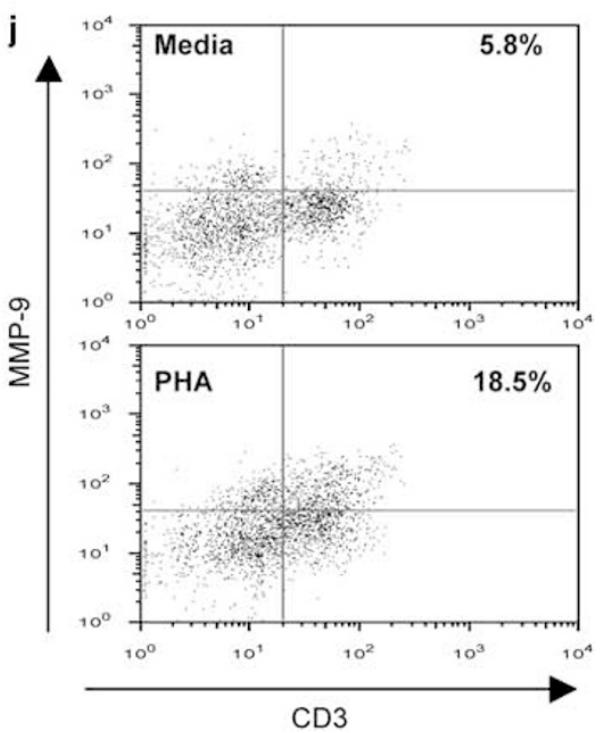




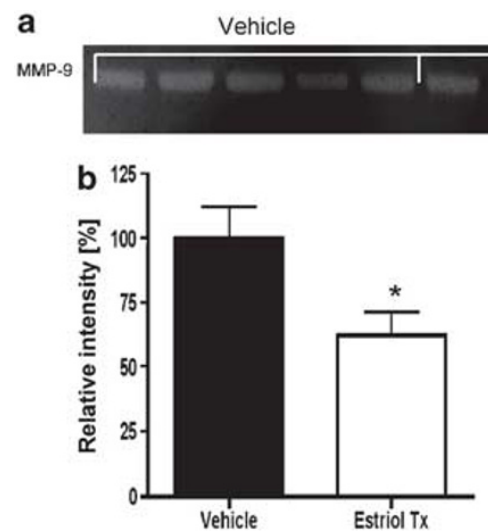

Estriol Tx

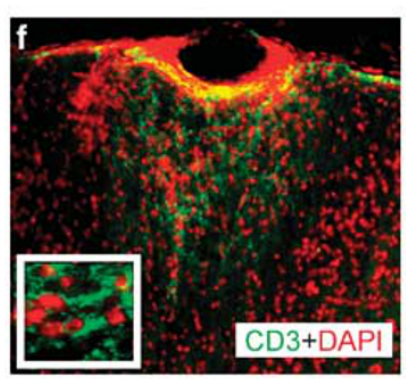

Vehicle

\section{Estriol Tx}
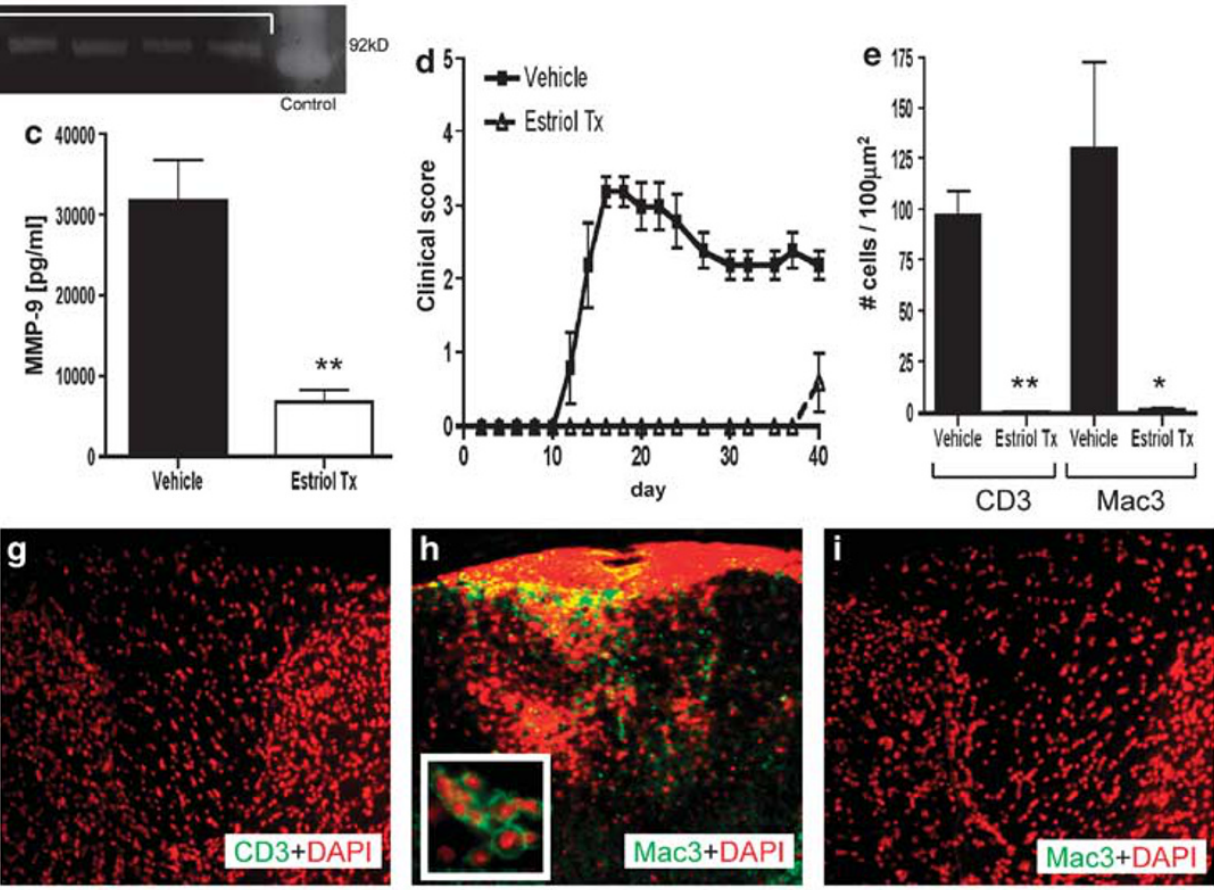

Estriol Tx

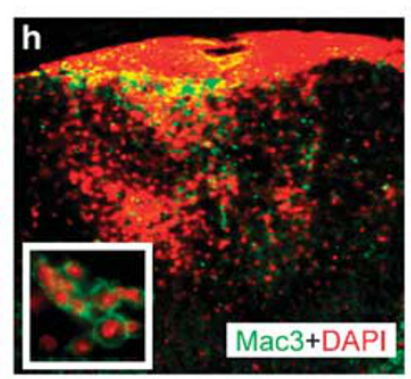

Vehicle

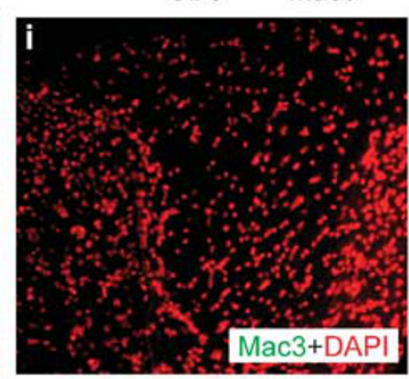

Estriol Tx

Figure 2 Estriol regulation of MMP-9 in active experimental autoimmune encephalomyelitis (EAE). Autoantigen-stimulated splenocyte cultured supernatants from estriol-treated EAE mice showed decreased MMP-9 bioactivity (a, quantification in b) as measured by zymography. Band intensity measurements were normalized by setting the mean band intensity of the vehicle-treated group as $100 \%$. Values are expressed as relative intensity (\%) for all animals. Estriol-treated animals also showed significantly reduced MMP-9 protein levels (c) compared to vehicle treated ( $n=5$ in each group). This was accompanied by abrogation of clinical disease (d). Estriol treatment decreased infiltration into the CNS by T cells (e). Representative images are shown in (f, g) (T cells are labeled with anti-CD3 antibodies, green staining). Similarly, estriol treatment decreased CNS infiltration by macrophages (e). Representative images are shown in (h, i) (macrophages are labeled with anti-Mac3 antibodies, green staining). The nuclear stain DAPI (pseudocolored red) was used to identify all cell nuclei. Images show dorsal column of spinal cord at $\times 10$ magnification (insets at $\times 40$ ).

\section{Decrease in MMP-9 in EAE is Mediated Through ER $\alpha$}

Effects of estrogens are mediated primarily by nuclear receptors $\operatorname{ER} \alpha$ and $\operatorname{ER} \beta$, which have distinct tissue distribution and function. Thus, we next sought to determine whether downregulation of MMP-9 by estriol treatment of EAE was mediated through $\operatorname{ER} \alpha$ or $\operatorname{ER} \beta$. To eliminate the effects of endogenous estrogens, female mice were ovariectomized in these experiments. Active EAE was induced in female C57BL/6 mice treated with optimal doses of a selective ER ligand for $\mathrm{ER} \alpha$ (PPT), ER $\beta$ (DPN) or with vehicle. Zymography showed decreased MMP-9 in autoantigen-stimulated splenocyte supernatants from $\operatorname{ER} \alpha(P<0.05)$, but not $\operatorname{ER} \beta$ ligandtreated EAE mice $(P>0.05)$ compared to vehicle treated (Figure $3 \mathrm{a}-\mathrm{c}$ ). This was confirmed by significant decreases in MMP-9 protein levels in splenocyte supernatant from ER $\alpha$ $(P<0.01)$, but not ER $\beta$ ligand $(P>0.05)$-treated EAE mice (Figure 3d).

Clinically, $\mathrm{ER} \alpha$ ligand treatment was associated with an early and complete abrogation of disease $(P<0.0001$; Figure $3 e)$. $\operatorname{ER} \beta$ ligand-treated mice, as compared with vehicletreated mice, were not significantly different early in disease (up to day 20 after disease induction) but then became significantly improved later during $\operatorname{EAE}(P<0.001)$. Immunohistochemistry analysis indicated decreased spinal cord infiltration by $\mathrm{T}$ cells (Figure $3 \mathrm{f}$, representative images shown in Figure $3 \mathrm{~g}$ and $\mathrm{h}$ ) and macrophages (Figure 3f, representative images shown in Figure $3 i$ and $j$ ) in $E R \alpha$ ligand $(P<0.05)$ treated but not ER $\beta$ ligand $(P>0.05)$-treated mice compared to vehicle-treated EAE mice.

\section{DISCUSSION}

In this study, we found that MMP-9 levels were decreased with estriol treatment at pregnancy doses in patients with RRMS. This decrease in MMP-9 coincided with a decrease in enhancing lesions on MRI. We then showed that MMP-9 levels were also decreased with estriol treatment at pregnancy doses in mice with EAE. Finally, we used this model to show that the estriol-mediated decrease in MMP-9 coincided with a decrease in $\mathrm{T}$ cell and macrophage infiltration into the CNS and that this decrease was mediated through ER $\alpha$.

Estrogens have a wide range of effects on the immune system that could be protective in EAE including down- 


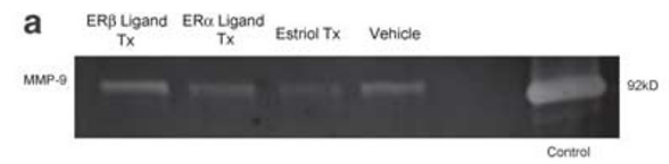

b ER $\beta$ Ligand Tx

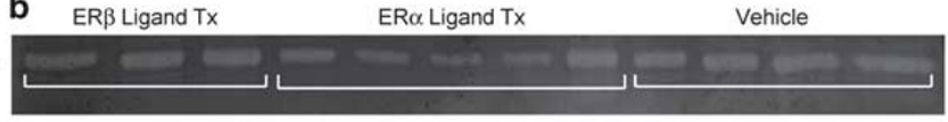

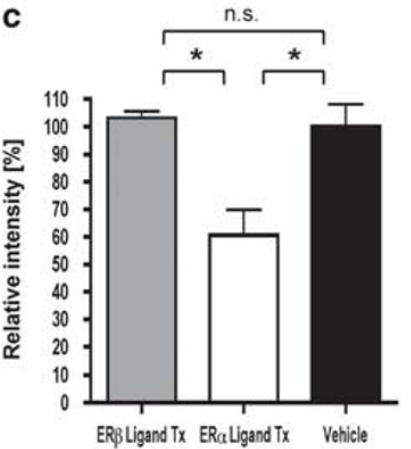

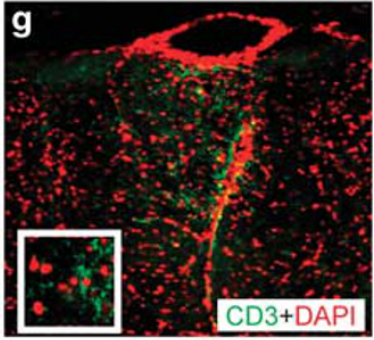

ER $\beta$ Ligand TX
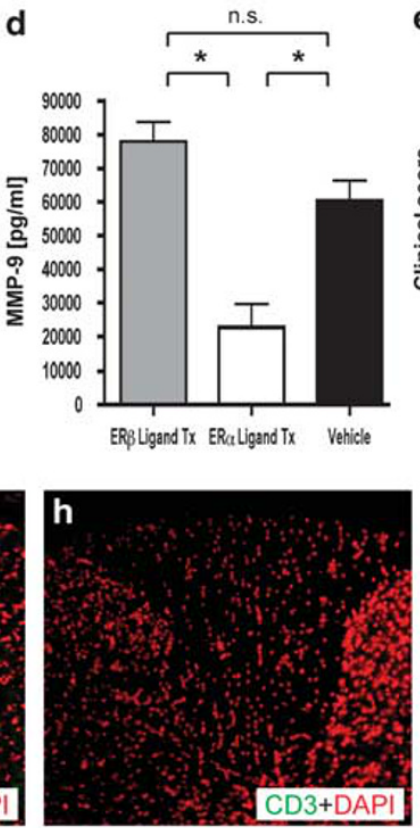

$E R \alpha$ Ligand Tx
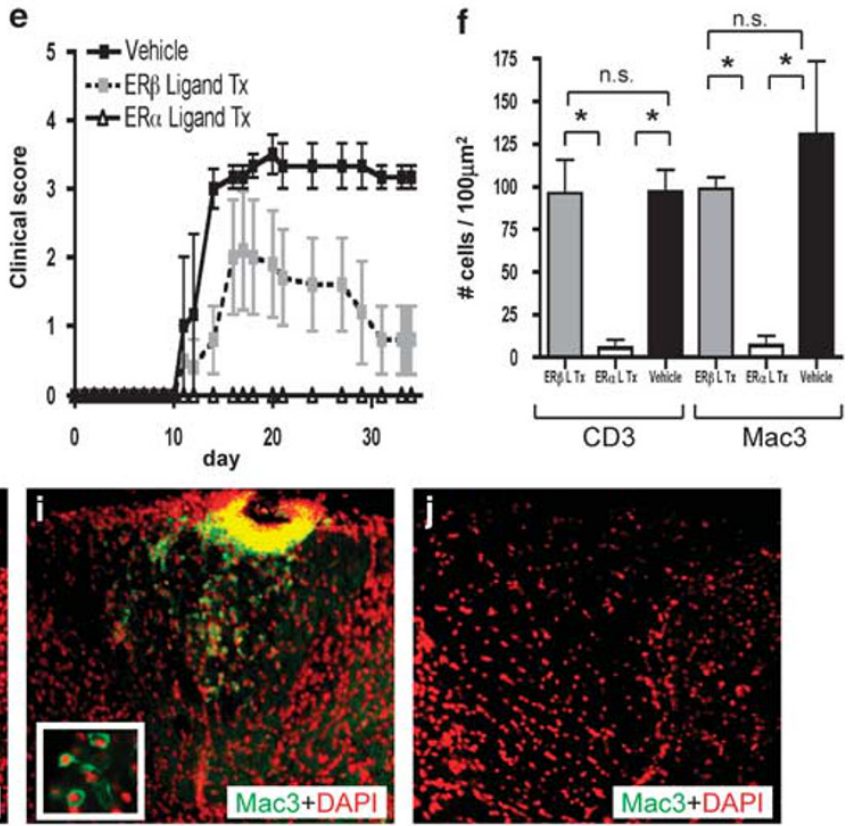

ER $\beta$ Ligand Tx

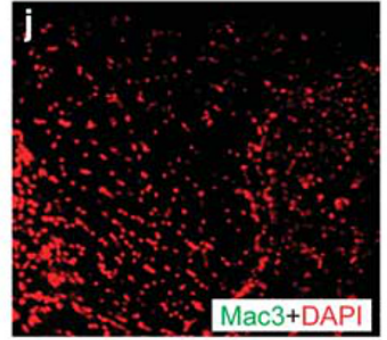

$E R \alpha$ Ligand Tx

Figure 3 Estriol-induced MMP-9 downregulation in EAE is mediated through ER $\alpha$. Decreased bioactivity of MMP-9 in supernatants from splenocyte cultures compared to vehicle treated was seen in ER $\alpha$ ligand and estriol-treated mice, but not in vehicle- or ER $\beta$ ligand-treated mice (a pooled supernatants from four animals in each group; b supernatants from individual animals; c quantification). Band intensity measurements were normalized by setting the mean band intensity of the vehicle-treated group as $100 \%$. Values are expressed as relative intensity (\%) for all animals. Significantly decreased MMP-9 protein levels were observed in ER $\alpha$ ligand-treated EAE mice $(n=5)$ compared to vehicle-treated EAE $(n=4)$, whereas there was no difference between vehicle treated and $\operatorname{ER} \beta$ ligand $(n=3)$-treated mice (d). ER $\alpha$ treatment completely abrogated clinical disease (e). ER $\alpha$, but not ER $\beta$, ligand treatment reduced infiltration into the CNS by T cells (f). Representative images are shown in (g, h) (T cells are labeled with anti-CD3 antibodies, green staining). Similarly, ER $\alpha$, but not ER $\beta$, ligand treatment decreased macrophages infiltration (f). Representative images are shown in (i, j) (macrophages are labeled with anti-Mac3 antibodies, green staining). The nuclear stain DAPI (pseudocolored red) was used to identify all cell nuclei. Images show dorsal column of spinal cord at $\times 10$ magnification (insets at $\times 40$ ).

regulation of cytokines, chemokines, dendritic cell function and induction of regulatory $\mathrm{T}$ cells. ${ }^{12}$ As estrogens are endogenous regulators of a great number of biological functions, a wide range of effects in the immune system is expected and no single mechanism is likely to be solely responsible for the protective effects in autoimmune disease. In the context of MS and EAE, MMP-9 may be of importance because it has a key role in transmigration of immune cells into the CNS, which is an early step in our current understanding of MS pathogenesis. MMP-9 is selectively expressed on Th1 cells compared with Th2 cells and is responsible for a higher migratory capacity of Th1 cells. ${ }^{19}$ Human monocytes have also been found to express high levels of MMP-9, which was linked to their migratory capacity in experimental models of the BBB. ${ }^{20}$ The importance of MMP-9 in autoimmune inflammation is further underscored by a study showing that GM6001, a matrix metalloproteinase inhibitor, ameliorated the clinical severity of EAE and reduced BBB disruption. $^{21}$
MMP-9 has been shown to be decreased by other immunomodulators such as IFN- $\beta^{22,23}$ and minocycline. ${ }^{24}$ Both drugs are also highly effective in reducing enhancing lesions on MRI by approximately $80 \%$ within the first 3 months of treatment. ${ }^{25,26}$ In contrast, there is no evidence to date that glatiramer acetate (GA) affects MMPs. ${ }^{27}$ Interestingly, GA does not significantly decrease enhancing lesions on serial MRI until month 6-9 of treatment. ${ }^{28}$ The ability of a drug to decrease MMP-9 thus seems to be linked to its ability to mediate an early and robust reduction in enhancing lesions. Our earlier report showing that estriol can decrease enhancing lesions by approximately $80 \%{ }^{15}$ is consistent with our finding herein that estriol treatment reduces MMP-9.

The synthetic estrogen ethinyl estradiol has been shown to decrease TNF- $\alpha$ as well as MMP-9 activity both in the peripheral immune system and the CNS in EAE, ${ }^{29}$ which is in line with our observation of decreased MMP-9 during treatment with pregnancy doses of estriol. Studies using ER $\alpha$ signaling deficient mouse strains have shown that clinical 
protection from EAE by estradiol ${ }^{30}$ and estriol ${ }^{31}$ depends on signaling through ER $\alpha$. Correspondingly, anti-inflammatory mechanisms of estrogens have been found to be mediated by $\mathrm{ER} \alpha$. We have reported earlier that treatment with a selective $\mathrm{ER} \alpha$ ligand, but not with an ER $\beta$ ligand, has immunomodulatory effects on peripheral cytokine production and reduces CNS infiltration. ${ }^{18}$ In addition to these peripheral immune effects, Garidou et $a l^{32}$ have elegantly shown that $\mathrm{ER} \alpha$-mediated regulation of resident CNS cells is important for amelioration of EAE. In another study, estradiol decreased MMP-9 production by microglia in $\mathrm{ER} \beta \mathrm{KO}$, but not $\mathrm{ER} \alpha \mathrm{KO}$, mice in a model of non-autoimmune CNS inflammation. ${ }^{33}$ Together, these results and our findings suggest that estrogens may interfere with CNS infiltration by inhibiting MMP-9 both within the periphery and the CNS in an $\mathrm{ER} \alpha$-dependent manner.

Interestingly, $\mathrm{ER} \alpha$ ligand treatment abrogated $\mathrm{EAE}$ at the onset and throughout the disease course, whereas $\operatorname{ER} \beta$ ligand treatment had no effect at disease onset but promoted recovery during the chronic phase of the disease. The late improvement of disability in $\mathrm{ER} \beta$-treated mice is in accordance with our earlier published study. ${ }^{18}$ There, we showed that though ER $\beta$ ligand treatment had no effects on splenocyte cytokine production and CNS inflammation, it reduced demyelination and preserved axon numbers in white matter, as well as decreased neuronal abnormalities in gray matter. This suggests a directly neuroprotective mechanism of $\operatorname{ER} \beta$ agonists independent of anti-inflammatory effects. These findings are in line with other recent studies using transgenic mice ${ }^{34}$ and selective ER $\beta$ agonists, ${ }^{35}$ indicating that the beneficial effects of estrogen on cognitive function are dependent on the $\operatorname{ER} \beta$ pathway. Selective $\operatorname{ER} \beta$ agonist effects on cognition have been linked to increased dendritic branching and upregulation of key synaptic proteins including PSD-95, synaptophysin and AMPA-receptor subunit, GluR1, in the hippocampus. ${ }^{36}$ To date, the exact mechanisms how $\operatorname{ER} \beta$ neuroprotection in EAE occurs, however, remain unclear and are the subject of ongoing studies.

Matrix metalloproteinases, and most importantly MMP-9, have a central role for term labor when expression and activation of MMP-9 increases during parturition. ${ }^{37}$ Elevated levels of MMP-9 have been implicated in several preterm perinatal complications including spontaneous preterm labor, premature rupture of fetal membranes and preeclampsia. ${ }^{37}$ Thus, MMP activity is tightly regulated during pregnancy. MS patients as well as individuals with other inflammatory autoimmune diseases such as rheumatoid arthritis (RA), uveitis and psoriasis experience clinical improvement during pregnancy, with a temporary 'rebound' exacerbation postpartum. ${ }^{8,38-43}$ MMP-9 could represent a common mechanism for immune cell homing in all of these disorders regardless of the target tissue of the autoimmune attack because levels of MMP-9 have been found to be elevated in RA, ${ }^{44}$ psoriasis ${ }^{44}$ and uveitis, ${ }^{45,46}$ in particular in patients with active disease. In psoriasis, effective therapy with anti-TNF- $\alpha$ was associated with decreased levels of MMP-9 in serum and skin lesions. ${ }^{47}$ These findings suggest that MMP-9 downregulation may be a shared mechanism that could underlie the decrease in disease activity of inflammatory autoimmune disorders such as MS, RA, uveitis and psoriasis during pregnancy.

This paper has focused on a potential beneficial effect of downregulation of MMP-9 during estriol treatment to decrease immune cell infiltration into the target tissue in autoimmune disease, however, other effects of MMP-9 that could have a role in MS pathology should also be considered. For example, high levels of MMPs in the CNS can contribute to demyelination and toxicity to axons. ${ }^{48}$ On the other hand, there is also emerging evidence that MMPs may be important for mediation of tissue repair. ${ }^{1}$ The regulation of these multiple and complex mechanisms during pregnancy and estriol treatment warrants further investigation.

\section{ACKNOWLEDGEMENTS}

The authors thank Dr Richard Olmstead for assistance with the statistical analyses. This work is supported by the NIH (RO1 NS45443) and the National Multiple Sclerosis Society (Grants RD3407, CA 1028 and FG 1702-A-1).

\section{DISCLOSURE/CONFLICT OF INTEREST}

The authors declare no conflict of interest.

1. Yong VW. Metalloproteinases: mediators of pathology and regeneration in the CNS. Nat Rev Neurosci 2005;6:931-944.

2. Lindberg RL, De Groot $\mathrm{CJ}$, Montagne $\mathrm{L}$, et al. The expression profile of matrix metalloproteinases (MMPs) and their inhibitors (TIMPs) in lesions and normal appearing white matter of multiple sclerosis. Brain 2001;124:1743-1753.

3. Lichtinghagen $R$, Seifert $T$, Kracke $A$, et al. Expression of matrix metalloproteinase- 9 and its inhibitors in mononuclear blood cells of patients with multiple sclerosis. J Neuroimmunol 1999;99:19-26.

4. Waubant E, Goodkin DE, Gee L, et al. Serum MMP-9 and TIMP-1 levels are related to MRI activity in relapsing multiple sclerosis. Neurology 1999;53:1397-1401.

5. Waubant E, Goodkin D, Bostrom A, et al. IFNbeta lowers MMP-9/TIMP-1 ratio, which predicts new enhancing lesions in patients with SPMS. Neurology 2003;60:52-57.

6. Lee MA, Palace J, Stabler G, et al. Serum gelatinase B, TIMP-1 and TIMP2 levels in multiple sclerosis. A longitudinal clinical and MRI study. Brain 1999;122:191-197.

7. Correale J, Bassani Molinas Mde L. Temporal variations of adhesion molecules and matrix metalloproteinases in the course of MS. J Neuroimmunol 2003;140:198-209.

8. Confavreux $C$, Hutchinson M, Hours MM, et al. Rate of pregnancyrelated relapse in multiple sclerosis Pregnancy in Multiple Sclerosis Group [see comments]. N Engl J Med 1998;339:285-291.

9. Kim S, Liva SM, Dalal MA, et al. Estriol ameliorates autoimmune demyelinating disease: implications for multiple sclerosis. Neurology 1999;52:1230-1238.

10. Palaszynski KM, Liu H, Loo KK, et al. Estriol treatment ameliorates disease in males with experimental autoimmune encephalomyelitis: implications for multiple sclerosis. J Neuroimmunol 2004;149:84-89.

11. Bebo Jr BF, Fyfe-Johnson A, Adlard K, et al. Low-dose estrogen therapy ameliorates experimental autoimmune encephalomyelitis in two different inbred mouse strains. J Immunol 2001;166:2080-2089.

12. Straub RH. The complex role of estrogens in inflammation. Endocr Rev 2007;28:521-574.

13. Ram $M$, Sherer $Y$, Shoenfeld $Y$. Matrix metalloproteinase- 9 and autoimmune diseases. J Clin Immunol 2006;26:299-307. 
14. Soldan SS, Retuerto Al, Sicotte NL, et al. Immune modulation in multiple sclerosis patients treated with the pregnancy hormone estriol. J Immunol 2003;171:6267-6274.

15. Sicotte NL, Liva SM, Klutch R, et al. Treatment of multiple sclerosis with the pregnancy hormone estriol. Ann Neurol 2002;52:421-428.

16. Zang YC, Halder JB, Hong J, et al. Regulatory effects of estriol on T cell migration and cytokine profile: inhibition of transcription factor NF-kappa B. J Neuroimmunol 2002;124:106-114.

17. Morales LB, Loo KK, Liu HB, et al. Treatment with an estrogen receptor alpha ligand is neuroprotective in experimental autoimmune encephalomyelitis. J Neurosci 2006;26:6823-6833.

18. Tiwari-Woodruff S, Morales LB, Lee R, et al. Differential neuroprotective and antiinflammatory effects of estrogen receptor (ER) $\{$ alpha $\backslash\}$ and ERbeta ligand tr. Proc Natl Acad Sci USA 2007;104:14813-14818.

19. Abraham M, Shapiro S, Karni A, et al. Gelatinases (MMP-2 and MMP-9) are preferentially expressed by Th1 vs Th2 cells. J Neuroimmunol 2005;163:157-164.

20. Bar-Or A, Nuttall RK, Duddy M, et al. Analyses of all matrix metalloproteinase members in leukocytes emphasize monocytes as major inflammatory mediators in multiple sclerosis. Brain 2003;126:2738-2749.

21. Gijbels K, Galardy RE, Steinman L. Reversal of experimental autoimmune encephalomyelitis with a hydroxamate inhibitor of matrix metalloproteases. J Clin Invest 1994;94:2177-2182.

22. Leppert $D$, Waubant $E$, Burk MR, et al. Interferon beta-1b inhibits gelatinase secretion and in vitro migration of human T cells: a possible mechanism for treatment efficacy in multiple sclerosis. Ann Neurol 1996;40:846-852.

23. Stuve O, Dooley NP, Uhm JH, et al. Interferon beta-1b decreases the migration of $\mathrm{T}$ lymphocytes in vitro: effects on matrix metalloproteinase-9. Ann Neurol 1996;40:853-863.

24. Brundula V, Rewcastle NB, Metz LM, et al. Targeting leukocyte MMPs and transmigration: minocycline as a potential therapy for multiple sclerosis. Brain 2002;125:1297-1308.

25. Metz LM, Zhang $Y$, Yeung $M$, et al. Minocycline reduces gadoliniumenhancing magnetic resonance imaging lesions in multiple sclerosis. Ann Neurol 2004;55:756.

26. Stone LA, Frank JA, Albert PS, et al. Characterization of MRI response to treatment with interferon beta-1b: contrast-enhancing MRI lesion frequency as a primary outcome measure. Neurology 1997;49:862869.

27. Yong VW, Zabad RK, Agrawal S, et al. Elevation of matrix metalloproteinases (MMPs) in multiple sclerosis and impact of immunomodulators. J Neurol Sci 2007;259:79-84.

28. Comi G, Filippi M, Wolinsky JS. European/Canadian multicenter, double-blind, randomized, placebo- controlled study of the effects of glatiramer acetate on magnetic resonance imaging-measured disease activity and burden in patients with relapsing multiple sclerosis. European/Canadian Glatiramer Acetate Study Group. Ann Neurol 2001;49:290-297.

29. Subramanian S, Matejuk A, Zamora A, et al. Oral feeding with ethinyl estradiol suppresses and treats experimental autoimmune encephalomyelitis in SJL mice and inhibits the recruitment of inflammatory cells into the central nervous system. J Immunol 2003;170:1548-1555.

30. Polanczyk M, Zamora A, Subramanian S, et al. The protective effect of 17 beta-estradiol on experimental autoimmune encephalomyelitis is mediated through estrogen receptor-alpha. Am J Pathol 2003;163:1599-1605.

31. Liu HB, Loo KK, Palaszynski K, et al. Estrogen receptor alpha mediates estrogen's immune protection in autoimmune disease. J Immunol 2003;171:6936-6940.

32. Garidou L, Laffont S, Douin-Echinard V, et al. Estrogen receptor alpha signaling in inflammatory leukocytes is dispensable for 17betaestradiol-mediated inhibition of experimental autoimmune encephalomyelitis. J Immunol 2004;173:2435-2442.

33. Vegeto $E$, Belcredito $S$, Etteri $S$, et al. Estrogen receptor-alpha mediates the brain antiinflammatory activity of estradiol. Proc Natl Acad Sci USA 2003;100:9614-9619.

34. Rissman EF, Heck AL, Leonard JE, et al. Disruption of estrogen receptor beta gene impairs spatial learning in female mice. Proc Natl Acad Sci USA 2002;99:3996-4001.

35. Rhodes ME, Frye CA. ERbeta-selective SERMs produce mnemonicenhancing effects in the inhibitory avoidance and water maze tasks. Neurobiol Learn Mem 2006;85:183-191.

36. Liu F, Day M, Muniz LC, et al. Activation of estrogen receptor-beta regulates hippocampal synaptic plasticity and improves memory. Nat Neurosci 2008;11:334-343.

37. Cockle JV, Gopichandran N, Walker JJ, et al. Matrix metalloproteinases and their tissue inhibitors in preterm perinatal complications. Reprod Sci 2007; 14:629-645.

38. Abramsky O. Pregnancy and multiple sclerosis. Ann Neurol 1994;36:S38-S41.

39. Birk K, Ford C, Smeltzer S, et al. The clinical course of multiple sclerosis during pregnancy and the puerperium. Arch Neurol 1990;47:738-742.

40. Da Silva JA, Spector TD. The role of pregnancy in the course and aetiology of rheumatoid arthritis. Clin Rheumatol 1992;11:189-194.

41. Damek DM, Shuster EA. Pregnancy and multiple sclerosis. Mayo Clin Proc 1997;72:977-989.

42. Nelson JL, Hughes KA, Smith AG, et al. Remission of rheumatoid arthritis during pregnancy and maternal-fetal class II alloantigen disparity. Am J Reprod Immunol 1992;28:226-227.

43. Runmarker B, Andersen O. Pregnancy is associated with a lower risk of onset and a better prognosis in multiple sclerosis [see comments]. Brain 1995;118:253-261.

44. Gruber BL, Sorbi D, French DL, et al. Markedly elevated serum MMP-9 (gelatinase B) levels in rheumatoid arthritis: a potentially useful laboratory marker. Clin Immunol Immunopathol 1996;78: 161-171.

45. Di Girolamo N, Verma MJ, McCluskey PJ, et al. Increased matrix metalloproteinases in the aqueous humor of patients and experimental animals with uveitis. Curr Eye Res 1996;15:1060-1068.

46. El-Shabrawi YG, Christen WG, Foster SC. Correlation of metalloproteinase- 2 and -9 with proinflammatory cytokines interleukin-1b, interleukin-12 and the interleukin-1 receptor antagonist in patients with chronic uveitis. Curr Eye Res 2000;20:211-214.

47. Cordiali-Fei P, Trento E, D'Agosto G, et al. Effective therapy with antiTNF-alpha in patients with psoriatic arthritis is associated with decreased levels of metalloproteinases and angiogenic cytokines in the sera and skin lesions. Ann N Y Acad Sci 2007;1110:578-589.

48. Yong VW, Giuliani $F$, Xue $M$, et al. Experimental models of neuroprotection relevant to multiple sclerosis. Neurology 2007:68:S32-S37 discussion S43-54. 